\title{
A distributional argument for supply-side climate policies
}

\author{
Geir B. Asheim*
}

July 9, 2012

\begin{abstract}
This paper presents a distributional argument for the use of supply-side climate policies whereby carbon emissions are controlled through (i) depletion quotas or (ii) permanent confiscation of a fraction of the in situ carbon stocks. The modeling considers intertemporal competitive equilibria in the Cobb-Douglas version of the Dasgupta-Heal-Solow-Stiglitz model of capital accumulation and costless resource extraction. It is shown how policies (i) and (ii) preserve the functional distribution of income between capital owners and resource owners, compared to the case where no climate policy is needed, while suggested demand-side policies do not. Such observations are of interest as avoiding functional redistribution may facilitate climate change negotiations. The paper discusses policy implications of the analysis outside the simplified setting of the stylized model.
\end{abstract}

Keywords and Phrases: Climate change negotiations, supply-side climate policies, functional income distribution, non-renewable resources

JEL Classification Numbers: Q54, D33, Q30

${ }^{*}$ I am grateful for valuable discussions with Steffen Kallbekken on the topic of this paper, many helpful suggestions from Bård Harstad, Michael Hoel and three anonymous referees, and comments received at presentations at VU University Amsterdam, the NORKLIMA workshop Oslo and the International Forum on Global Agreements on Environmental Protection and Sustainability at the University of Exeter. This research has received funding from Frisch Centre project 3181 and been supported by l'Institut d'études avancées - Paris. The paper is also part of the research activities at the Centre for the Study of Equality, Social Organization, and Performance (ESOP) at the Department of Economics at the University of Oslo. ESOP is supported by the Research Council of Norway.

Address: Department of Economics, University of Oslo, P.O. Box 1095 Blindern, NO-0317 Oslo, Norway. Tel: +47 22855498 Fax: +47 22855035 Email: g.b.asheim@econ.uio.no 


\section{Introduction}

Anthropogenic climate change is in large measure caused by release to the atmosphere of carbon from stable deposits in underground reservoirs of fossil fuels and in forests formed through photosynthesis. With the exception of recent attempts to avoid or delay tropical deforestation, the UNFCCC's emphasis on territorial emissions has lead to a process focused on demand-side efforts that seek to control emissions of carbon dioxide to the atmosphere through emission quotas or emission taxes. This paper presents a distributional argument for the use of supply-side policies whereby carbon emissions are controlled through depletion quotas or through permanent confiscation of a fraction of the in situ carbon stocks, and argues that such instruments may facilitate international climate change negotiations.

Without major advances in CCS, successful climate policies rely on limiting the cumulative emissions of carbon and require that large deposits of coal and unconventional oil and gas be left in situ for the indefinite future. One possible policy measure, in the spirit of the initiatives to protect tropical forests, is to buy up property rights for in situ fossil fuels which, taking into account the long-run costs and risks of climate change, should be left undeveloped and put them in a permanent reserve. These are the deposits for which extraction has the smallest economic value and the highest environmental cost.

Another possibility is to let net carbon depletion be controlled through depletion quotas or depletion taxes. In comparison to emission quotas or emission taxes, two advantages are clear:

(i) The number of agents that deplete carbon from deposits (or are potential participants in CCS efforts) is much smaller than the number of agents that emit carbon dioxide to the atmosphere (see, however, the assessment of Aldy et al., 2010, sect. 3.1.1).

(ii) If climate policies succeed and the world becomes less reliant on burning carbon, then the institutions can be rolled back, in particular if implemented through long-term depletion quotas allocated to a small number of actual and potential fossil fuel producers. In contrast, they need to be maintained when policies are based on demand-side instruments, if negligible carbon-use remains wide-spread.

The supply-side perspective and the fact that greenhouse gas emissions to a large extent derive from depleting non-renewable resources have gained much prominence in 
the recent debate on the "green paradox" initiated by Sinn (2008) and further discussed in many recent papers (see, e.g., Gerlagh, 2011; Hoel, 2010, 2012; van der Ploeg and Withagen, 2011). This literature points out that development of backstop technologies and escalation of carbon pricing may constitute a gradual confiscation of stocks of nonrenewable resources and, hence, may motivate their owners to accelerate the extraction of such resources. As a byproduct, this increases the rate of carbon emissions. Climate policies that seek to develop backstop technologies and escalate carbon pricing may thus have perverse consequences. In the present paper I consider such supply-side effects in a very simple setting as, in my context, the avoidance of unacceptable damage from climate change makes the resource stock valueless.

Supply-side instruments have also been discussed in the academic literature, with Bohm (1993) and Hoel (1994) being early contributions and Harstad (2012a,b) representing recent ones, as well as in more popularized writings (Tickell, 2008). One motivation for this literature has been to investigate whether supply-side policies have better properties than demand-side policies when the free-riding of some set of countries leads to partial participation. Throughout most of the present paper I assume full participation and note the distributional consequences of different kinds of policies. Such observations are of interest as avoiding functional redistribution may be a prerequisite for climate agreements with wide participation and may thus facilitate climate change negotiations. However, I also point out that supply-side policies may be less prone to leakage if participation is not global.

My modeling entails that resource extraction is costless up to total exhaustion. This implies that a large portion of the carbon taxation (or other kinds of carbon pricing) designed to reduce cumulative resource extraction is borne by the resource owners as the net (of carbon taxation) price of resource input is pushed down to zero. In the real world, where different deposits of fossil fuels have different extraction costs, the incidence of carbon taxation will be more moderate (see, e.g. Méjean and Hope, 2010).

I have adopted the perspective that the atmosphere's cumulative capacity for absorbing $\mathrm{CO}_{2}$ is a non-renewable and exhaustible resource. In the medium run, in which oceans and other carbon sinks contribute to the absorption of atmospheric carbon, this simplification is not accurate. However, in the very long run and with the motivation that serious climate change should not only be delayed but even avoided, this perspective is relevant and supported by climate modeling (cf. Allen et al., 2009).

In the present paper I investigate the distributional effects of climate policies by considering intertemporal competitive equilibria within the Cobb-Douglas version of 
the Dasgupta-Heal-Solow-Stiglitz (DHSS) model of capital accumulation and costless resource extraction (Dasgupta and Heal, 1974; Solow, 1974; Stiglitz, 1974). Since the assumption of no extraction cost implies that the resource stock is homogeneous, there is no problem of selecting which resources are to be put into permanent reserve. Moreover, I will assume that announced policies are perceived to be credible; e.g., it is believed that resources put into permanent reserve will remain unavailable for development and extraction at some later point in time.

In the setting of this stylized model I will show how permanent confiscation, without compensation, of a fraction of the in situ carbon resources large enough to avoid serious climate change preserves the functional distribution of income between capital owners and resource owners. Furthermore, I show that this solution can be obtained also by depletion quotas, but not by other suggested alternatives.

As the DHSS abstracts from many features of the real world, the presentation of this paper should be interpreted as pedagogical device intended to stimulate discussion by illustrating distributional consequences of different kinds of climate policies. In the interest of clarity, I have kept my model as simple as possible (instead of introducing complicating features like those considered by Eisenack et al., 2012). Even though the introduction of backstop technologies and the division of fossil fuels into different extraction cost classes would modify the conclusions considerably, I still believe that the illustrations presented within the setting of the DHSS model might be of interest.

In Section 2 I introduce the model and present as a benchmark closed-form solution for the optimal paths of production, consumption, net investment and resource depletion, without and with the potential for climate change damage. In Section 3, I analyze the distribution consequences of demand-side instruments, while in Section 4, I do the same for supply-side instruments. In Section 5, I illustrate the results within the context of a two-country world. Finally, I discuss the relevance of these observations in Section 6 and offer concluding remarks in Section 7.

\section{Model}

Consider the Cobb-Douglas version of the DHSS model:

$$
Q=K^{\alpha} R^{\beta} N^{1-\alpha-\beta}=C+\dot{K},
$$

where I denote by $Q$ non-negative net production, by $K$ non-negative capital, by $R$ non-negative resource input (of fossil fuel), by $N$ positive population which is assumed 
to be constant, and by $C$ non-negative consumption, and where

$$
\alpha>0, \beta>0, \alpha+\beta<1 .
$$

The assumption that $\alpha+\beta<1$ means that labor inputs are productive. There is no depreciation of capital and no cost of extracting the resource.

Let the lower-case variables, $q, c, k, r$, refer to per capita values so that

$$
q=k^{\alpha} r^{\beta}=c+\dot{k},
$$

as the production function exhibits constant returns to scale and population is constant.

There are positive initial per capita stocks of capital and resource, $\left(k_{0}, m_{0}\right) \gg 0$. To model climate change as simply as possible, assume that there is a cumulative absorption capacity in per capita terms, $\bar{m}>0$, of emitted resource input, up to which climate change causes no damage and above which climate change causes unacceptable damage. One might interpret $\bar{m}$ as an agreed-upon target for cumulative emission; however, if there are damages also below $\bar{m}$, then consumption ceases to be an indicator of wellbeing and the paths that I consider will not be optimal.

The path $\{c(t), q(t), k(t), r(t)\}_{t=0}^{\infty}$ is feasible if

$$
\int_{0}^{\infty} r(t) d t \leq \min \left\{m_{0}, \bar{m}\right\}
$$

are satisfied, and (1) holds for a.e. $t>0$ with $k(0)=k_{0}$. Henceforth, $a$ 'path' will always refer to a feasible path. In the deterministic setting considered here, if $\bar{m}<m_{0}$ so that the climate threshold $\bar{m}$ is binding, then the resource stock $m(t)=m_{0}-\int_{0}^{t} r(\tau) d \tau$ can be depleted down to $m_{0}-\bar{m}$ without incurring any risk for climate change damage. Note that this modeling of climate change in the setting of the DHSS model entails that greenhouse gas emission is in fixed proportions to resource input, and the only form for mitigation is to reduce resource input.

I will present closed-form solutions of paths with sustainable consumption, defined as paths for which consumption never exceeds the sustainable level. For this purpose, I assume that an efficient path with a constant savings rate $s$ is chosen; that is, at all times, $\dot{k}(t)=s q(t)$. As shown by Asheim et al. (2007, Thm. 10), ${ }^{1}$ (i) if $s=\beta$, then the efficient constant savings rate path is optimal according to a maximin objective, so that $\{c(t), q(t), k(t), r(t)\}_{t=0}^{\infty}$ satisfies

$$
\inf _{t \geq 0} c(t) \geq \inf _{t \geq 0} \tilde{c}(t)
$$

\footnotetext{
${ }^{1}$ With stationary population, the savings rate $s$ is both a gross of population growth savings rate and a net of population growth savings rate, which are denoted $a$ and $b$ in Asheim et al. (2007) respectively.
} 
for any path $\{\tilde{c}(t), \tilde{q}(t), \tilde{k}(t), \tilde{r}(t)\}_{t=0}^{\infty}$, and (ii) if $s \in(\beta, \alpha)$, then the efficient constant savings rate path is optimal according to an undiscounted utilitarian objective with constant elasticity of marginal utility given by

$$
\eta=\frac{1-\beta}{s-\beta},
$$

so that $\{c(t), q(t), k(t), r(t)\}_{t=0}^{\infty}$ satisfies

$$
\limsup _{T \rightarrow \infty}\left(\int_{0}^{T} \frac{\tilde{c}(t)^{1-\eta}}{1-\eta} d t-\int_{0}^{T} \frac{c(t)^{1-\eta}}{1-\eta} d t\right) \leq 0
$$

for any path $\{\tilde{c}(t), \tilde{q}(t), \tilde{k}(t), \tilde{r}(t)\}_{t=0}^{\infty}$. An efficient constant savings rate path with $s \geq \alpha$ is not feasible, and an efficient constant savings rate path with $s<\beta$ is not sustainable.

Eqs. (10), (12) and (17)-(24) of Asheim et al. (2007) imply that the efficient constant savings rate path with $s \in[\beta, \alpha)$ corresponds to the following closed-form solution:

$$
\begin{gathered}
c(t)=(1-s) q(t), \\
q(t)=q(0)\left(1+\sigma \frac{q(0)}{k_{0}} t\right)^{\frac{s}{\sigma}}-1, \\
k(t)=k_{0}\left(1+\sigma \frac{q(0)}{k_{0}} t\right)^{\frac{s}{\sigma}}, \\
r(t)=\left(q(0) k_{0}^{-\alpha}\right)^{\frac{1}{\beta}}\left(1+\sigma \frac{q(0)}{k_{0}} t\right)^{-\frac{\alpha-s}{\sigma}-1},
\end{gathered}
$$

where

$$
q(0)=\left[(\alpha-s)^{\beta} k_{0}^{\alpha-\beta}\left(\min \left\{m_{0}, \bar{m}\right\}\right)^{\beta}\right]^{\frac{1}{1-\beta}}
$$

and $\sigma:=[(1-\alpha-\beta) s+\alpha \beta] /(1-\beta)$. In the maximin case, $\beta=s=\sigma$, so that consumption, production and capital investment are constant, as established already by Solow (1974, sect. 9). In the undiscounted utilitarian case, $\sigma<s<\alpha$, so that consumption, production and capital investment are increasing functions of time; see Dasgupta and Heal (1979, pp. 303-308) for an early treatment of this case.

Asheim et al. (2007) provide closed-form solutions also for efficient constant savings rate paths with quasi-arithmetic growth in population and technology. The present analysis concentrates on efficient constant savings rate paths with stationary population and stationary technology only because these are the simplest sustainable paths. Everything can be generalized to the wider classes of sustainable paths considered by Asheim et al. (2007).

Let $p(t)$ denote the marginal productivity of resource input at time $t$ :

$$
p(t)=\beta \frac{q(t)}{r(t)} .
$$


The path described by (2)-(5) satisfies the Hotelling rule since the growth rate of $p(t)$ equals the marginal productivity of capital:

$$
\frac{\dot{p}(t)}{p(t)}=\alpha \frac{q(t)}{k(t)} .
$$

The Hotelling rule ensures no profitable arbitrage of resource input. If, on the one hand, $m_{0}<\bar{m}$, so that the resource constraint $m_{0}$ is binding, $p(t)$ is the Hotelling rent on the resource, while there is no scarcity rent on the cumulative absorption capacity. If, on the other hand, $\bar{m}<m_{0}$, so that the climate threshold $\bar{m}$ is binding, $p(t)$ is the scarcity rent on the cumulative absorption capacity, while the Hotelling rent on the resource is zero.

Since the Hotelling rule is satisfied, the path can be implemented as a decentralized intertemporal competitive equilibrium, with the savings rate $s$ determined endogenously, for appropriate intertemporal preferences for the consumers. In this equilibrium at time $t$, the wage rate equals the marginal productivity of labor, $(1-\alpha-\beta) q(t)$, the rate of interest equals the marginal productivity of capital, $\alpha q(t) / k(t)$, and the resource price equals $p(t)$. The possibility of decentralization relates to whether the path is competitive, which in this model corresponds to the Hotelling rule; not to whether the path is optimal according to a particular criterion (like discounted utilitarianism).

To be explicit, assume that the economy is divided into $I$ dynasties, where each dynasty $i$ has a constant population share $n^{i}$ and initial per capita endowments of capital $k_{0}^{i}$ and resource $m_{0}^{i}$. The initial endowments of the $I$ dynasties add up to the initial aggregate per capita stocks: $\sum_{i=1}^{I} k_{0}^{i}=k_{0}$ and $\sum_{i=1}^{I} m_{0}^{i}=m_{0}$.

Each dynasty $i$ manages its stocks of capital and resource to maximize a maximin objective in the case where $s=\beta$ and an undiscounted utilitarian objective with constant elasticity of marginal utility equal to $(1-\beta) /(s-\beta)$ in the case where $s \in(\beta, \alpha)$, subject to its initial endowments and its budget constraint at each time $t$. Since all dynasties are faced with the same structure of interest rates (and, in the case where $s>\beta$, the utility function has constant elasticity of marginal utility), their consumption paths will be scaled versions of the aggregate consumption path.

If, $m_{0}<\bar{m}$, so that the resource constraint $m_{0}$ is binding, then dynasty $i$ 's budget constraint at time $t$ is

$$
c^{i}(t)+\dot{k}^{i}(t)+p(t) \dot{m}^{i}(t)=(1-\alpha-\beta) q(t) n^{i}+\alpha q(t) \frac{k^{i}(t)}{k(t)} .
$$

The mix of $k^{i}(t)$ and $m^{i}(t)$ does not matter for each dynasty $i$, as the Hotelling rule ensures that both assets have the same return. At the aggregate level, the objectives of 
the dynasties and the intertemporal competitive equilibrium ensure that $\sum_{i=1}^{I} k^{i}(t)=$ $k(t)$ and $\sum_{i=1}^{I} m^{i}(t)=m(t)$ hold at each time $t$. Summing (B0) over all dynasties and invoking (7) yield $\sum_{i=1}^{I} c^{i}(t)+\dot{k}(t)-\beta q(t)=(1-\alpha-\beta) q(t)+\alpha q(t)$, showing by eq. (1) that the consumption of the dynasties add up to what is available at each time $t$.

I follow common terminology by letting the functional income distribution refer to the distribution of income between the owners of the various factors of production. I base my income definition on the theory of comprehensive accounting as developed since Weitzman's (1976) seminal article. In particular, I apply the Sefton-Weale (Sefton and Weale, 2006) accounting methodology (national income is the present value of the future interest on consumption) and its use by Asheim and Wei (2009) (sectoral income is the present value of the future interest on cash-flow accruing to the sector). By this national income definition, (i) welfare is increasing if and only if national income exceeds consumption, and (ii) national income equals net national product in a closed economy with stationary technology. Furthermore, it can be divided in a natural way into income accruing to individuals. The sectoral income definition splits national income on the various sectors by keeping track of how each sector contributes to individual income.

In the case of efficient constant savings rate paths in the Cobb-Douglas version of the DHSS model when no climate policy is needed (i.e., $m_{0}<\bar{m}$ ), it is particularly easy to derive the functional income shares (see Asheim and Hartwick, 2011, Sect. 7). Then total consumption $(1-s) q(t)$ (which equals total cash-flow) is divided into a cash-flow $(1-\alpha-\beta) q(t)$ of wages to workers, a cash-flow $(\alpha-s) q(t)$ of return on capital minus the cost of reinvestment to capital owners, and a cash-flow $\beta q(t)$ of revenue to resource owners. Because of the Cobb-Douglas technology and the constant savings rate, the relative shares of the total cash-flow accruing to the owners of the various factors of production are fixed through time. Therefore, these relative shares are the functional income shares, as summarized in the following table.

Table 0: Relative functional income shares in the DHSS model with no policy $\left(m_{0}<\bar{m}\right)$

\begin{tabular}{lc}
\hline Workers: & $\frac{1-\alpha-\beta}{1-s}$ \\
Capital owners: & $\frac{\alpha-s}{1-s}$ \\
Owners of resource (or cumulative absorption capacity): & $\frac{\beta}{1-s}$ \\
\hline
\end{tabular}

Even though the technology is stationary, the environments for the workers, the capital owners, and the resource owners are not stationary. The workers experience an increasing wage (if $s>\beta$ ), the capital owners a decreasing interest rate, and resource 
owners an increasing resource price. Thus, "terms-of-trade" improve for workers and in particular for resource owners, while they deteriorate for capital owners. This is reflected by the dynamic income definition that underlies Table 0. Furthermore, Table 0 can be used to determine the relative income share of each dynasty $i$ as follows:

$$
\frac{1-\alpha-\beta}{1-s} \cdot n^{i}+\frac{\alpha-s}{1-s} \cdot \frac{k^{i}(t)}{k(t)}+\frac{\beta}{1-s} \cdot \frac{m^{i}(t)}{m(t)} .
$$

The question posed in the next three sections is: What kind of climate policies leave the relative functional shares unchanged when comparing a situation where $m_{0}<\bar{m}$, so that the resource constraint $m_{0}$ is binding, with a situation where $\bar{m}<m_{0}$, so that the climate threshold $\bar{m}$ is binding and climate policies are needed to implement the optimal path?

An alternative approach would be to assume that $\bar{m}<m_{0}$ and compare the distributional effects of the inefficient solution where no policy is implemented with different kinds of efficient climate policies. However, to determine functional shares of dynamic income in the former case one must specify what would happen when accumulated resource extraction goes beyond $\bar{m}$, both in terms of the behavior of the path and in terms of climate damages. Moreover, such an analysis would involve the calculation of dynamic income in an economy with an imperfect resource allocation mechanism (Arrow et al., 2003), in a setting with a maximin or undiscounted utilitarian objective.

Keeping $m_{0}$ fixed, and comparing a situation where $\min \left\{m_{0}, \bar{m}\right\}=m_{0}$ with a situation where $\min \left\{m_{0}, \bar{m}\right\}<m_{0}$, one can also ask what happens to the absolute functional income shares. Since total income at time 0 equals net product $(=(1-$ $\beta) q(0))$ and $q(0)$ is given by (6), it follows that the elasticity of total income w.r.t. to $\bar{m}$ equals $\beta /(1-\beta)$ when the threshold $\bar{m}$ is binding. Since $\beta<\alpha<1-\beta$, this elasticity is less one, implying that a smaller $\bar{m}$ reduces income but less than proportionally.

In Sections 3 and 4 I assume that the policies are implemented by a global government, an assumption that will be relaxed in the subsequent Section 5 .

\section{Demand-side policies}

In the present section I consider demand-side climate instruments - emission taxes and emission quotas which can be auctioned off or allocated freely - in the case where $\bar{m}<m_{0}$, so that the climate threshold $\bar{m}$ is binding. Throughout I assume that tax revenues and revenues from quota sales accrue to the government. The path is efficient only if these revenues are distributed back to the consumers as lump-sum transfers, 
but I do not specify how this is done. Under the assumption that all consumers have the same intertemporal preferences, there are no general equilibrium effects of different distributional policies.

Policy 1: Emission taxes or auctioned emission quotas. Since $\bar{m}<m_{0}$, so that the path does not exhaust the resource stock, Hotelling rents are zero along the optimal path, and $p(t)$, as determined by (3), (5) and (7), is the scarcity rent on the cumulative absorption capacity. This in turn means that an emission tax in terms of resource input must equal $p(t)$ at each point in time to implement the optimal path. With this time path of emission taxes, the demand of resource input will be $r(t)$ at each point in time, leading to a cumulative extraction that approaches $\bar{m}$ asymptotically. Note that there are no profitable market opportunities for the remaining resource stock $m_{0}-\bar{m}$, even with zero extraction costs, as increased extraction of resource input would have lead to a negative price. As revenues from the emission tax are assumed to accrue to the government and resource owners receive zero revenues, the relative shares become:

\begin{tabular}{lc}
$\begin{array}{l}\text { Table 1: Distributional effects of Policy } \mathbf{1} \\
\text { Relative functional shares with emission } \\
\text { taxes or auctioned emission quotas }\end{array}$ \\
\hline Workers: & $\frac{1-\alpha-\beta}{1-s}$ \\
Capital owners: & $\frac{\alpha-s}{1-s}$ \\
Resource owners: & 0 \\
Government: & $\frac{\beta}{1-s}$ \\
\hline
\end{tabular}

Hence, the consequence of emission taxes in the simplified setting of the DHSS model is to confiscate the entire resource wealth and allocate the property rights to the cumulative absorption capacity, $\bar{m}>0$, of emitted resource input to the government.

In the case of auctioned emission quotas, the optimal emission quota in terms of resource input is, at each point in time, set equal to $r(t)$ as determined by (5) and auctioned off by the government. Since $\bar{m}<m_{0}$ and the equilibrium price of resource input is zero at each point in time, the equilibrium price for emission quotas in terms of resource input will equal $p(t)$ at each point in time. As the revenues from the auction of quotas are assumed to accrue to the government and resource owners receive zero revenues, the relative shares become the same as for emission taxes. Hence, also with auctioned emission quotas, the entire resource wealth is confiscated and and the property rights to the cumulative absorption capacity, $\bar{m}>0$, of emitted resource input are allocated to the government. 
Both emission taxes and auctioned emission quotas make dynasty $i$ 's resource stock $m^{i}(t)$ valueless, so that its $i$ 's budget constraint at time $t$ becomes:

$$
c^{i}(t)+\dot{k}^{i}(t)=(1-\alpha-\beta) q(t) n^{i}+\alpha q(t) \frac{k^{i}(t)}{k(t)}+g^{i}(t) .
$$

Here $g^{i}(t)$ denotes the lump-sum transfer at time $t$ from the government to dynasty $i$, with $\sum_{i=i}^{I} g^{i}(t)=p(t) r(t)=\beta q(t)$ being equal to the government's revenues from the emission taxes or auctioned emission quotas. This shows explicitly how the path is implemented as a decentralized intertemporal competitive equilibrium, provided that the dynasties have appropriate intertemporal preferences.

Policy 2: Freely allocated emission quotas. The optimal emission quota for resource input is set equal to $r(t)$ at each point in time and allocated freely to the capital owners (which are responsible for production and hence emissions). As for the case of auctioned quotas, the equilibrium price of resource input will be zero and the equilibrium price for emission quotas in terms of resource input in the quota market will equal $p(t)$ at each point in time. As the capital owners pay for neither resource input nor emission quotas, the relative shares become:

Table 2: Distributional effects of Policy 2

\section{Relative functional shares with} freely allocated emission quotas

\begin{tabular}{lc}
\hline Workers: & $\frac{1-\alpha-\beta}{1-s}$ \\
Capital owners: & $\frac{\alpha+\beta-s}{1-s}$ \\
Resource owners: & 0 \\
Government: & 0 \\
\hline
\end{tabular}

Hence, also in this case, the entire resource wealth is confiscated. However, in this case, the property rights to the cumulative absorption capacity, $\bar{m}>0$, of emitted resource input are allocated to the capital owners.

In this case, decentralization is achieved by letting dynasty $i$ 's budget constraint at time $t$ be given by

$$
c^{i}(t)+\dot{k}^{i}(t)=(1-\alpha-\beta) q(t) n^{i}+\alpha q(t) \frac{k^{i}(t)}{k(t)}+p(t) r(t) \frac{k_{0}^{i}}{k_{0}},
$$

implying that the emission quotas are grand-fathered to the capital owners according to their initial stocks. Such grand-fathering ensures that the dynasties manage their assets efficiently if emission quotas are made tradable. 
In all cases considered, the relative functional share of the resource owners is reduced from $\beta /(1-s)$ to 0 when going from the case where $m_{0}<\bar{m}$ to the case where $\bar{m}<m_{0}$, unless the government uses revenues from emission taxes or auctions of emission quotas to compensate their loss of revenue.

\section{Supply-side policies}

In the present section I consider supply-side climate instruments - depletion taxes and depletion quotas which can auctioned off or allocated freely - in the case where $\bar{m}<m_{0}$, so that the climate threshold $\bar{m}$ is binding. I also consider putting the fraction $m_{0}-\bar{m}$ of the resource into a permanent reserve, not to be depleted, with or without compensation to the resource owners.

Policy 3: Depletion taxes or auctioned depletion quotas. Since $\bar{m}<m_{0}$, so that the path does not exhaust the resource stock, Hotelling rents must be zero. This in turn means that an depletion tax in terms of resource input must equal $p(t)$ at each point in time. As these tax revenues are assumed to accrue to the government and resource owners receive zero revenues, the relative shares become:

Table 3: Distributional effects of Policy 3

Relative functional shares with depletion taxes or auctioned depletion quotas

\begin{tabular}{lc}
\hline Workers: & $\frac{1-\alpha-\beta}{1-s}$ \\
Capital owners: & $\frac{\alpha-s}{1-s}$ \\
Resource owners: & 0 \\
Government: & $\frac{\beta}{1-s}$ \\
\hline
\end{tabular}

Auctioned depletion quotas will have the same distributional effects: The optimal depletion quota for resource input is set equal to $r(t)$ at each point in time and auctioned off by the government at equilibrium price $p(t)$ at each point in time. Decentralization is achieved by letting dynasty $i$ 's budget constraint at time $t$ be given by (B1).

Hence, as for the corresponding demand policy (Policy 1), the consequence of depletion taxes or auctioned depletion quotas in the simplified setting of the DHSS model is to confiscate the entire resource wealth and allocate the property rights to the cumulative absorption capacity, $\bar{m}>0$, of emitted resource input to the government.

Policy 4: Freely allocated depletion quotas. The optimal depletion quota for resource input is set equal to $r(t)$ at each point in time and allocated freely to the resource 
owners. As for the case of auctioned quotas, the equilibrium price of resource input will be zero and the equilibrium price for depletion quotas for resource input in the quota market will equal $p(t)$ at each point in time. Since the depletion quotas are freely allocated to the resource owners, the revenue from the sale of resource input accrue to resource owners and the relative shares become:

\begin{tabular}{|c|c|}
\hline \multicolumn{2}{|c|}{$\begin{array}{l}\text { Relative functional shares with } \\
\text { freely allocated depletion quotas }\end{array}$} \\
\hline Workers: & $\frac{1-\alpha-\beta}{1-s}$ \\
\hline Capital owners: & $\frac{\alpha-s}{1-s}$ \\
\hline Resource owners: & $\frac{\beta}{1-s}$ \\
\hline Government: & 0 \\
\hline
\end{tabular}

Hence, in this case the property rights to the cumulative absorption capacity, $\bar{m}>0$, of emitted resource input is allocated to the resource owners, implying that the net confiscation of the resource stock is limited to the fraction $m_{0}-\bar{m}$ that will not be produced. The increased scarcity of the resource compensates the resource owners for the effective confiscation of this fraction and thus preserves the functional distribution of income between capital owners and resource owners.

In this case, decentralization is achieved by letting dynasty $i$ 's budget constraint at time $t$ be given by

$$
c^{i}(t)+\dot{k}^{i}(t)=(1-\alpha-\beta) q(t) n^{i}+\alpha q(t) \frac{k^{i}(t)}{k(t)}+p(t) r(t) \frac{m_{0}^{i}}{m_{0}},
$$

implying that the depletion quotas are grand-fathered to the resource owners according to their initial stocks. Such grand-fathering ensures that the dynasties manage their assets efficiently with and without tradable depletion quotas. Without tradable quotas, the dynasties will deplete their stocks at the same relative rate. Then each dynasty $i$ receives $p(t) r(t) m_{0}^{i} / m_{0}=-p(t) \dot{m}^{i}(t)$ in revenues from its quotas at each time $t$, and budget constraint (B4) can be seen to coincide with the budget constraint (B0) in the situation where the resource constraint $m_{0}$ is binding (i.e., $m_{0}<\bar{m}$ ).

Policy 5: Compensated confiscation of resource stock. The fraction of the resource stock $m_{0}-\bar{m}$ not to be depleted is purchased at market prices from the resource owners and put into a permanent reserve. It follows from the Hotelling rule that the time of purchase plays no role, as long as it is perceived that the purchase will take place 
at these terms and the compensation of the resource owners is at each point in time balanced by lump-sum taxes on the economy at large. The relative functional share accruing to the resource owners net of the compensation received equals $\beta /(1-s)$. When the compensation is added, this share is multiplied by $m_{0} / \bar{m}$, since the resource owners are paid not only for resources they deplete, but also for those that are put in a permanent reserve. The difference

$$
\frac{\beta}{1-s} \frac{m_{0}}{\bar{m}}-\frac{\beta}{1-s}
$$

is the purchase price (relative to total income) received from the government. Thus, the relative shares become:

$\begin{gathered}\text { Table 5: Distributional effects of Policy } \mathbf{5} \\
\text { Relative functional shares with com- }\end{gathered}$
\begin{tabular}{lc}
\multicolumn{1}{c}{ pensated confiscation of resource stock } \\
\hline Workers: & $\frac{1-\alpha-\beta}{1-s}$ \\
Capital owners: & $\frac{\alpha-s}{1-s}$ \\
Resource owners: & $\frac{\beta}{1-s} \frac{m_{0}}{\bar{m}}$ \\
Government: & $-\frac{\beta}{1-s} \frac{m_{0}-\bar{m}}{\bar{m}}$ \\
\hline
\end{tabular}

Hence, in this case the resource owners are not only allocated the property rights to the cumulative absorption capacity, $\bar{m}>0$, of emitted resource input, but they also receive compensation for the valueless part of the resource stock $m_{0}-\bar{m}>0$ at the higher scarcity rents induced by the stricter cumulative depletion constraint that the potential for climate change amounts to. Since the increased scarcity of the resource by itself compensates the resource owners for the loss of $m_{0}-\bar{m}$, this scheme in effect overcompensates the resource owners at the expense of the government. Since a smaller $\bar{m}$ reduces income but less than proportionally, it follows that the resource owners' income share is increased not only in relative terms, but also in absolute terms.

In this case, decentralization is achieved by letting dynasty $i$ 's budget constraint at time $t$ be given by

$$
\begin{aligned}
c^{i}(t) & +\dot{k}^{i}(t)+p(t) \dot{m}^{i}(t) \\
& =(1-\alpha-\beta) q(t) n^{i}+\alpha q(t) \frac{k^{i}(t)}{k(t)}+p(t) r(t) \frac{m_{0}-\bar{m}}{\bar{m}} \frac{m_{0}^{i}}{m_{0}}-\left(-g^{i}(t)\right) .
\end{aligned}
$$

Here $-g^{i}(t)$ denotes dynasty $i$ 's lump-sum tax to the government at time $t$, with

$$
\sum_{i=i}^{I}\left(-g^{i}(t)\right)=p(t) r(t) \frac{m_{0}-\bar{m}}{\bar{m}}=\beta q(t) \frac{m_{0}-\bar{m}}{\bar{m}},
$$


so that tax revenues balance the government's compensation of the resource owners.

Policy 6: Uncompensated confiscation of resource stock. The fraction of the resource stock $m_{0}-\bar{m}$ not to be depleted is confiscated from the resource owners and put into a permanent reserve. As the increased scarcity of the resource compensates the resource owners for the confiscation of this fraction, the functional distribution of income between capital owners and resource owners is preserved:

\begin{tabular}{|c|c|}
\hline & \\
\hline Workers: & $\frac{1-\alpha-\beta}{1-s}$ \\
\hline Capital owners: & $\frac{\alpha-s}{1-s}$ \\
\hline Resource owners: & $\frac{\beta}{1-s}$ \\
\hline Government: & 0 \\
\hline
\end{tabular}

Hence, this policy, together with the case of freely allocated depletion quotas, are the only two that preserve the functional distribution of income when going from a situation where $m_{0}<\bar{m}$, so that the resource constraint $m_{0}$ is binding, to a situation where $\bar{m}<m_{0}$, so that the climate threshold $\bar{m}$ is binding. Decentralization is achieved by letting dynasty $i$ 's budget constraint at time $t$ be given by (B0).

\section{A two-country world}

The merits of demand-side and supply-side policies can be investigated further by relaxing the assumption that there is a global government able to implement an efficient policy. Instead, I consider a two-country world, where competitive world markets with perfect capital mobility and free trade in resource input and the consumption good ensure overall productive efficiency in the absence of climate damage.

I make the following assumptions: The total population is divided equally between the two countries, so that the population size of each equals $\frac{1}{2} N$. Residents of country 1 own the entire capital stock. There is no labor mobility, but perfect capital mobility. So, since both countries are assumed to have access to the same technology, half of the capital stock is employed in each country to ensure equal capital-labor ratio and thereby efficiency. Hence, under such efficient allocation of capital, production is divided equally between the countries and thus emissions are divided equally between the countries. 
Table 7: Functional income shares in a two-country world

\begin{tabular}{lcccccc}
\hline \hline Policy & \multicolumn{2}{c}{ Country 1} & \multicolumn{3}{c}{ Country 2} \\
& $\begin{array}{c}\text { Capital- } \\
\text { owners }\end{array}$ & $\begin{array}{c}\text { Resource- } \\
\text { owners }\end{array}$ & $\begin{array}{c}\text { Govern- } \\
\text { ment }\end{array}$ & $\begin{array}{c}\text { Capital- } \\
\text { owners }\end{array}$ & $\begin{array}{c}\text { Resource- } \\
\text { owners }\end{array}$ & $\begin{array}{c}\text { Govern- } \\
\text { ment }\end{array}$ \\
\hline \hline 0 NoPol $\left(m_{0}<\bar{m}\right)$ & $\frac{\alpha-s}{1-s}$ & 0 & 0 & 0 & $\frac{\beta}{1-s}$ & 0 \\
\hline 1 EmTax/AucEmQ & $\frac{\alpha-s}{1-s}$ & 0 & $\frac{1}{2} \frac{\beta}{1-s}$ & 0 & 0 & $\frac{1}{2} \frac{\beta}{1-s}$ \\
2 FreeEmQ & $\frac{\alpha+\beta-s}{1-s}$ & 0 & 0 & 0 & 0 & 0 \\
\hline 3 DepTax/AucDepQ & $\frac{\alpha-s}{1-s}$ & 0 & 0 & 0 & 0 & $\frac{\beta}{1-s}$ \\
4 FreeDepQ & $\frac{\alpha-s}{1-s}$ & 0 & 0 & 0 & $\frac{\beta}{1-s}$ & 0 \\
5 CompConfisc & $\frac{\alpha-s}{1-s}$ & 0 & $-\frac{1}{2} \frac{\beta}{1-s} \frac{m_{0}-\bar{m}}{\bar{m}}$ & 0 & $\frac{\beta}{1-s} \frac{m_{0}}{\bar{m}}$ & $-\frac{1}{2} \frac{\beta}{1-s} \frac{m_{0}-\bar{m}}{\bar{m}}$ \\
6 UncompConfisc & $\frac{\alpha-s}{1-s}$ & 0 & 0 & 0 & $\frac{\beta}{1-s}$ & 0 \\
\hline \hline
\end{tabular}

Population is equal to $\frac{1}{2} N$ in each country, so that capital and resource input is divided equally between the countries. The entire capital stock is owned by residents of country 1 . The entire resource stock is located in country 2 and owned by residents of country 2. Policy 0 is no policy intervention. Policies 1-2 are supply-side policies as described in Section 3, with revenues from emission taxes and emission quotas divided in proportion to emissions. Policies 3-6 are demand-side policies as described in Section 4 , with revenues from depletion taxes and depletion quotas divided in proportion to extraction. Policy 5 entails that resource owners are compensated according to Lindahl pricing (cf. Harstad, 2012a, sect. IV.D).

Residents of country 2 own the entire resource stock. As, of course, the in situ resource stock is not mobile, all extraction of resource input takes place in country 2 , while half of the flow of resource input is employed in each country.

Table 7 summarizes the distributional effects of the six policies considered in Sections 3 and 4 . For all six policies, the aggregate path is given by (2)-(5) under the assumption that $\bar{m}<m_{0}$, so that the climate threshold $\bar{m}$ is binding and climate policies are needed to implement the optimal path.

The relative functional income shares are compared to the relative functional income shares in a hypothetical situation where $m_{0}<\bar{m}$, so that the resource constraint $m_{0}$ would be binding (corresponding to Policy 0 in Table 7 ). The table shows that only the supply-side policies whereby resource depletion is controlled through freely allocated depletion quotas (Policy 4) or through permanent and uncompensated confiscation of a fraction of resource stock (Policy 6 ) lead to unchanged distribution when moving from a situation with $m_{0}<\bar{m}$ to a situation with $\bar{m}<m_{0}$.

In Table $7 \mathrm{I}$ have assumed that the revenues from emission or depletion taxes and from auctioning emission or depletion quotas accrue to the country where the emission or depletion occurs. Because capital, although being owned solely by inhabitants of country 1, is employed in equal measure in each country, half of the revenues from 
emissions taxes or auctions of emission quota accrue to each country. The resource stock, in contrast, is assumed to be immobile, so that all revenues from depletion taxes or auctions of depletion quotas accrue to country 2. This implies that, in addition to Policies 4 and 6, also Policy 3 (depletion taxes or auctioned depletion quotas) leaves the international distribution of income unchanged when climate policies are introduced.

Up to now I have assumed full participation and compliance, so that both countries participate in a climate agrement and undertake the agreed upon measures. Is there any difference in the performance of the various policies if there is only partial participation or compliance? In the setting of this section this means that only one of the two countries implements climate policies.

If only one country, say country $i$, implements a demand-side policy like Policy 1 , then capital - assumed to be mobile - will relocate to the country, say country $j$, not implementing climate policies to equalize returns to investment. Moreover, resource input will leak in the direction of country $j$, and the differential in real wage between the countries will provide an incentive for migration in the same direction. Hence, a unilateral demand-side policy that increases the price for carbon emissions in one country only will lead to major inefficiencies, but will have no effect on the eventual cumulative depletion of the resource stock. It will therefore not be able to ensure that the climate threshold $\bar{m}$ is observed. The conclusion is that a one-country demand-side policy is wasteful, and has no long run climate effect.

The situation is very different if only country 2 implements supply-side policies $3-4$ or 6 . Since the entire resource stock is located in country 2, this country can on its own implement the optimal policy without changing the relative distribution of income between the two countries. Of course, in the spirit of the Coase-conjecture (Coase, 1972), country 2 will be tempted to announce such policies and then renege later when cumulative depletion is getting close to the climate threshold $\bar{m}$. However, if we abstract from this question of credibility, the conclusion is that a one-country supply-side policy is optimal if implemented by the country endowed with the resource.

For both capital and the resource, I have assumed that the whole stock is owned by residents of one country (or one part of the world) only. So equally extreme assumptions are made for both capital and the resource. The fundamental difference is that capital is assumed to be perfectly mobile, while in situ resources are assumed to be perfectly immobile. Hence, it is sufficient to use supply-side policies for the part of the world endowed with resources, while demand-side policies must have universal coverage if the factors of production are mobile and all locations have access to the same technology. 


\section{Discussion}

The analysis of the previous sections has on distributional grounds pointed towards a solution where all of the resource is confiscated, but where the property rights to the atmosphere as a carbon sink is awarded to the resource owners by allowing them to produce a fraction of the total in situ stock.

The analysis has been performed in the setting of the DHSS model and, as indicated already in the introduction, this model constitutes a far too simple representation of real-word resource markets. Extraction costs differ greatly between, on the one hand, easily available oil in major Middle-East fields and, on the other hand, marginal deposits of oil, gas and coal, including unconventional sources of fossil fuels.

If policies designed to avoid unacceptable climate change damage effectively enforce a ceiling on the cumulative extraction of fossil fuels, then cost efficiency demands that the deposits for which extraction has the smallest economic value and the highest environmental cost are left unextracted. Simply confiscating such marginal deposits will be at the expense of countries in which these resources are located, and to the benefit of countries - like Saudi Arabia - with low-extraction-cost resources, since the enhanced scarcity that such policies induce will increase resource rents for deposits from which the policies allow extraction. Indeed, Persson et al. (2007) find that major oil producers may profit rather than lose even if demand-side climate policies are used, since low-extraction-cost oil producers may receive higher producer prices (i.e., prices net of emission taxes or the cost of emission quotas) in spite of the policies. Likewise, freely allocating depletion quotas in a cost efficient manner will in effect mimic the behavior of a comprehensive and internationally sanctioned and monitored OPEC, where the original OPEC members will come out as winners. ${ }^{2}$

Still, one should be concerned about the futility of demand-side policies:

(i) The UNFCCC process has attempted to implement demand-side efforts that, if successful, would limit the cumulative extraction of fossil fuel by lowering the producer prices of such fuels, and seeks to combine this with redistribution towards the developing world via the CDM or the Green Climate Fund. Attempting to take property rights away from resource owners at the same time as externalities are sought to be internalized might be a recipe for inaction. The reason is that without agreement on property rights there is no basis on which a mutually beneficial bargain on climate policies might be

\footnotetext{
${ }^{2}$ When fossil fuel production is controled by a cartel, one has to analyze how climate policies interact with cartel behavior; see Liski and Tahvonen (2004). Such analysis is outside the scope of this paper.
} 
reached. The attempts of resource-endowed countries like Saudi Arabia and Venezuela to sabotage the UNFCCC process illustrate the roadblocks that this might lead to.

(ii) Partial demand-side policies lead to leakage of carbon to non-committed countries, also from sources in countries that in principle support the demand-side policies. The result may well be that world-wide deposits of coal are extracted and transported to China as input for industrial production of manufactured goods which are re-exported back to the countries where the coal originated (see, e.g., Rosenthal, 2010). Unless demand-side policies are supported by all actual or potential industrial powers (or a system of border tax adjustments is put in place), such efforts will not be effective in reducing the eventual cumulative extraction of fossil fuels. In contrast, partial supplyside policies may well be effective, even if the situation is not as simple as the one described in Section 5.

The main asymmetry between demand-side policies and supply-side policies is caused by the mobility of capital (and labor) and the immobility of in situ resources. This means that demand-side policies must include all actual and potential output producing countries, while supply-side policies must include only countries with actual carbon resources. In line with Harstad's (2012a) analysis, it is actually sufficient to include the countries where the medium-extraction-cost carbon resources that should be put in permanent reserve are located. One need not be concerned with low-extractioncost carbon resources that should be produced also under efficient climate policies or high-extraction-cost carbon resources that will not be produced even if no policy is implemented. However, countries with low-extraction-cost carbon resources might find in their own interest to compensate the owners of the medium-extraction-cost carbon resources that efficient supply-side policies keep off the market, as this limits the cumulative supply of fossil fuels.

In this paper I have not analyzed the effects of future advances in (i) CCS and (ii) new backstop technologies that may serve as potential low cost future sources of energy and fuel. If CCS - carbon capture and storage - is successful in establishing stable deposits of $\mathrm{CO}_{2}$, then, within the context of the supply-side policies having been discussed here, such efforts can be credited with depletion quotas which may be sold to owners of fossil fuel deposits.

Will technological progress ensure that enough carbon is left indefinitely in stable underground reservoirs to prevent unacceptable climate change damage without using explicit instruments to limit extraction? This may be the case if the developed new techniques lead to greater reductions in the costs of producing energy from alternative 
(non-fossil fuel) sources than in the costs of producing energy by extracting marginal and unconventional sources of fossil fuels. However, as illustrated by the recent technological progress in the production of shale gas, it is not assured that technological progress will be directed towards non-fossil fuel backstop technologies. Hence, it seems reasonable to assume that development of backstop technologies will serve as a complement to, but not a substitute for, more direct efforts designed to reduce the eventual cumulative extraction of fossil fuels.

\section{Concluding remarks}

The analysis of this paper has emphasized advantages of supply-side climate policies within the simplified setting of the DHss model. The subsequent discussion has pointed to reasons why this stylized model may not be an adequate representation of the real world. In this concluding section I discuss issues that must be resolved in order to implement a supply-side policy that seeks to leave the functional distribution unchanged.

(a) Selection. With different classes of extraction costs, medium-extraction-cost carbon resources should be left in situ and put in permanent reserve. As illustrated by the recent technological progress in the production of shale gas, it is hard to predict what medium-extraction-cost deposits should be kept in indefinite reserve. This is an argument for a flexible system that allows for future re-evaluation.

(b) Cost. The owners of such medium-extraction-cost in situ fossil fuels can (at least in part) be compensated by countries with low-extraction-cost carbon resources, as these countries gain as as a consequence of the resulting enhanced scarcity rent for fossil fuels.

(c) Control. The authority enforcing that in situ fossil fuels are kept in permanent reserve must be respected by the host country. This amounts to preventing the host country access to these resources, which can be achieved even by threat of physical force.

(d) Credibility. One must avoid that (it is believed that) resources put into permanent reserve will be available for development and extraction at some later point in time, because otherwise current prices for fossil fuels will not be affected. Destroying the resources will make them permanently inaccessible, but also reduce flexibility in the event future technological progress changes relative extraction costs. This is an argument for, instead, seeking to design policies that are timeconsistent (cf. Harstad, 2012a, sect. IV.B). 
This discussion goes beyond the analysis of the stylized model presented this paper and it is therefore speculative. The analysis and the discussion might, however, motivate a renewed debate on the need for a re-orientation of climate policies.

\section{References}

Aldy, J.E., Krupnick, A.J., Newell, R.G., Parry, I.W.H., Pizer, W.A. (2010). Designing climate mitigation policy. Journal of Economic Literature 48, 903-934.

Allen, M.R., Frame, D.J., Huntingford, C., Jones, C.D., Lowe, J.A., Meinshausen, M., Meinshausen, N. (2009). Warming caused by cumulative carbon emissions towards the trillionth tonne. Nature 458, 1163-1166.

Arrow, K., Dasgupta, P.S., and Mäler, K.-G. (2003). Evaluating projects and assessing sustainable development in imperfect economies. Environmental and Resource Economics 26, $647-685$.

Asheim, G.B., Buchholz, W., Hartwick, J.M., Mitra, T., Withagen, C. (2007). Constant savings rates and quasi-arithmetic population growth under exhaustible resource constraints. Journal of Environmental Economics and Management 53, 213-229.

Asheim, G.B., Hartwick, J.M. (2011). Anomalies in green national accounting. Ecological Economics 70, 2303-2307.

Asheim, G.B., Wei, T. (2009). Sectoral income. Environmental and Resource Economics 42, $65-87$.

Bohm, P. (1993). Incomplete international cooperation to reduce $\mathrm{CO}_{2}$ emissions: Alternative policies. Journal of Environmental Economics and Management 24, 258-271.

Coase, R. (1972). Durability and monopoly. Journal of Law and Economics 15, 143-49.

Dasgupta, P.S., Heal, G.M. (1974). The optimal depletion of exhaustible resources. Review of Economic Studies (Symposium), 3-28.

Dasgupta, P.S., and Heal, G.M. (1979), Economic Theory and Exhaustible Resources. Cambridge University Press, Cambridge, UK.

Eisenack, K., Edenhofer, O., Kalkuhl, M. (2012). Energy taxes, resource taxes and quantity rationing for climate protection. Forthcoming in Energy Policy, DOI: 10.1016/ j.enpol.2012.05.001

Gerlagh, R. (2011). Too much oil. CESifo Economic Studies 57, 79-102. 
Harstad, B. (2012a). Buy coal! A case for supply-side environmental policy. Journal of Political Economy 120, 77-115.

Harstad, B. (2012b). The market for conservation and other hostages. Mimeo, MEDS, Kellogg School, Northwestern University.

Hoel, M. (1994). Efficient climate policy in the presence of free riders. Journal of Environmental Economics and Management 27, 259-274.

Hoel, M. (2010). Is there a Green Paradox? CESifo Working Paper No. 3168.

Hoel, M. (2012). Carbon taxes and the green paradox. In Climate Change and Common Sense: Essays in Honour of Tom Schelling, R.W. Hahn, A. Ulph (eds). Oxford: Oxford University Press.

Liski, M., Tahvonen, O. (2004). Can carbon tax eat OPECs rents? Journal of Environmental Economics and Management 47, 1-12.

Méjean, A., Hope, C. (2010). The effect of $\mathrm{CO}_{2}$ pricing on conventional and non-conventional oil supply and demand. Cambridge Working Paper in Economics 1054.

Persson, T.A., Azar, C., Johansson, D., Lindgren, K. (2007). Major oil exporters may profit rather than lose, in a carbon-constrained world. Energy Policy 35, 6346-6353.

van der Ploeg, F., Withagen, C. (2011). Is there really a green paradox? Forthcoming in Journal of Environmental Economics and Management.

Rosenthal, E. (2010). Nations that debate coal use export it to feed China's need. New York Times, November 21, 2010. Available at http://www.nytimes.com/2010/11/22/science/ earth/22fossil.html

Sefton, J., Weale, M. (2006). The concept of income in a general equilibrium. Review of Economic Studies 73, 219-249.

Sinn, H.W. (2008). Public policies against global warming. International Tax and Public Finance 15, 360-94.

Solow, R.M. (1974). Intergenerational equity and exhaustible resources. Review of Economic Studies (Symposium), 29-45.

Stiglitz, J. (1974). Growth with exhaustible natural resources: Efficient and optimal growth paths. Review of Economic Studies (Symposium), 123-137.

Tickell, O. (2008). Kyoto2: How to Manage the Global Greenhouse. London: Zed Books.

Weitzman, M.L. (1976). On the welfare significance of national product in a dynamic economy, Quarterly Journal of Economics 90, 156-162. 